12

\title{
Изотопное отношение испаряемых ионов, критические расстояния ионизации и зоны ионизации в процессе полевого испарения молибдена при высоких температурах
}

\author{
(C) О.Л. Голубев, Н.М. Блашенков
}

Физико-технический институт им. А.Ф. Иофрфе РАН, 194021 Санкт-Петербург, Россия

e-mail: O.Golubev@mail.ioffe.ru

(Поступило в Редакцию 1 ноября 2016 г.)

С помощью магнитного масс-спектрометра с полевым источником ионов изучалось стационарное полевое испарение молибдена при температурах $1000-2000 \mathrm{~K}$. В процессе испарения наблюдались ионы всех семи

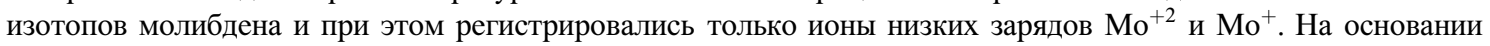
измеренных энергий ионов, а также и определенной экспериментально величины напряженности испаряющего поля были определены критические расстояния ионизации и зоны ионизации для одно- и двухзарядных ионов Мо. Показано, что образование ионов в процессе полевого испарения атомов поверхности происходит на некоторых расстояниях от поверхности эмиттера в очень узкой пространственной зоне.

DOI: 10.21883/JTF.2017.08.44733.2089

\section{Введение}

Явление полевого испарения состоит в том, что поверхностные атомы эмиттера испаряются в виде положительных ионов только под воздействием сильного электрического поля напряженностью $F$ порядка нескольких десятков вольт на нанометр (обычно $F \sim 10-60 \mathrm{~V} / \mathrm{nm})$, известно это явление довольно давно [1]. Для создания сильного испаряющего электрического поля обычно используются эмиттеры-катоды из проводящего материала в виде острий с радиусом закругления в конце острия $r \sim 50-150 \mathrm{~nm}$, к аноду же прикладывается высокое стационарное напряжение $U \sim 10-15 \mathrm{kV}$. Впервые вопрос об определении расстояния от поверхности эмиттера, на котором происходит образование иона из атома, был поставлен при изучении процесса полевой ионизации газов, т.е. образования ионов из нейтральных атомов или молекул газов вблизи поверхности проводящего (как правило, металлического) эмиттера под действием только сильного электрического поля. В присутствии сильного электрического поля при приближении атома к поверхности эмиттера его энергия возрастает, и для ионизации необходимо, чтобы уровень электрона в атоме располагался выше уровня Ферми в металле. При приближении к поверхности прозрачность барьера для электрона повышается и, наконец, при достижении некоего критического расстояния $x_{\mathrm{cr}}$ энергия уровня электрона в атоме становится равной энергии Ферми в металле, в этом случае возможен туннельный переход электрона в металл и образование иона. Необходимо отметить, что величина $x_{\mathrm{cr}}-$ это не просто расстояние, на котором ионизуется атом, а минимально возможное расстояние от поверхности, на котором ионизация еще возможна, и при этом процесс ионизации может происходить только в пределах некой весьма узкой пространственной зоны $\Delta$ вблизи поверхности эмиттера. Обычно значения величин $x_{\mathrm{cr}}$ для большинства газов лежат в пределах $0.46-0.60 \mathrm{~nm}$, а ширина зоны, в которой происходит ионизация, очень мала, как правило, ее значения составляют $\Delta \sim 0.015-0.030 \mathrm{~nm}$. Зона ионизации узка, потому что ближе $x_{\mathrm{cr}}$ атом ионизоваться не может, поскольку энергетический уровень электрона в атоме становится ниже уровня Ферми в металле, и переход электрона в металл невозможен, а удаление атома от поверхности приводит к быстрому падению прозрачности потенциального барьера и вследствие этого к очень сильному падению вероятности туннельной ионизации [2].

Иная ситуация возникает в случае полевого испарения, когда ионы образуются за счет испарения и ионизации атомов самого острия-эмиттера. В случае классического полевого испарения при криогенных температурах ионы бывают многозарядными, и испаряющие поля достигают очень больших величин $F_{\mathrm{ev}} \sim 50-60 \mathrm{~V} / \mathrm{nm}$, и в этом случае значения $x_{\text {cr }}$ могут оказаться меньше радиуса атома. Авторы практически единственной корректной работы [3] пытались экспериментально определить величину $x_{\text {cr }}$ по скорости полевого испарения вольфрама, и эта величина при небольшой скорости испарения $10^{-2}$ моноатомного слоя в секунду в поле $F_{\mathrm{ev}}=57 \mathrm{~V} / \mathrm{nm}$ оказалась весьма малой: $x_{\mathrm{cr}} \sim 0.13 \mathrm{~nm}$, т.е. меньше условного радиуса атома вольфрама $r=0.143 \mathrm{~nm}$. Вследствие этого принято было считать, что величина $x_{\mathrm{cr}}$ не имеет в случае полевого испарения реального смысла и испарение атома в виде иона происходит непосредственно с поверхности эмиттера. Однако если использовать полевое испарение при достаточно высоких температурах эмиттера, то ситуация становится принципиально иной. Особенностями полевого испарения при высоких температурах являются резкое понижение заряда испаряемого 
иона с ростом температуры вследствие заметного снижения величин $F_{\mathrm{ev}}$, а также появление заметной величины энергии активации процесса испарения $Q_{n}$ вместо практически нулевых ее значений в случае испарения при криогенных температурах [4-6]. Если величины $Q_{n}$ уже не равны нулю, то и значения $x_{\text {cr }}$ (будем называть эту величину критическим расстоянием, просто следуя традиции) также могут быть с нашей точки зрения более значительными.

Следовательно, главной задачей настоящей работы является определение расстояний от поверхности эмиттера $x_{\text {cr }}$, на которых происходит превращение атома в ион в процессе полевого испарения Мо при высоких температурах, а также величин зон $\Delta$ вблизи поверхности эмиттера, внутри которых происходит процесс ионизации.

\section{Экспериментальные результаты и обсуждение}

Эксперименты проводились, главным образом, с эмиттерами из Мо с использованием полевого магнитного масс-спектрометра. Этот прибор представлял собой статический магнитный секторный масс-спектрометр, который обладал разрешением по массе $\delta m / m \sim 1 / 200$ и уникальным полевым источником ионов. Источник этот имел острие-эмиттер ионов и соответствующую систему линз и щелей: характеристики данного источника подробно описаны в работе [4]. Указанного разрешения по массам вполне хватало для уверенного наблюдения на масс-спектрах сигналов от всех 7 изотопов Мо с массами от $\mathrm{Mo}^{92}$ до $\mathrm{Mo}^{100}$. Вакуум в нашем приборе был не слишком высоким, как правило, на уровне $p \sim 10^{-8}$ torr, однако это не имело принципиального значения, поскольку эксперименты проводились при достаточно высоких температурах эмиттера $T \sim 1000-2000 \mathrm{~K}$.

Как уже отмечалось, в случае полевого испарения при криогенных температурах наблюдаются ионы высоких зарядов, молибден испаряется в основном в виде ионов $\mathrm{Mo}^{+3}$, меньше наблюдается ионов $\mathrm{Mo}^{+2}$ и совсем мало ионов $\mathrm{Mo}^{+4}$. Однако если проводить процесс полевого испарения при достаточно высоких $T$, то ситуация становится принципиально иной. Нами был проведен ряд исследований полевого испарения некоторых металлов и сплавов при $T \geq 1000 \mathrm{~K}$ [5-7]. Основными особенностями процесса испарения при этом были, во-первых, резкое понижение количества ионов высоких зарядов и увеличение количества испаряемых низкозарядных ионов с ростом $T$, а во-вторых - сильные флуктуации ионного тока. В нашем случае испарения Мо при комнатной температуре наблюдались в основном ионы $\mathrm{Mo}^{+3}$, а при $T \geq 1000 \mathrm{~K}$ наблюдались только ионы $\mathrm{Mo}^{+}$и $\mathrm{Mo}^{+2}$, причем если до $T \sim 1400 \mathrm{~K}$ преобладали двухзарядные ионы, то при более высоких $T$ ионный ток состоял уже в основном из однозарядных ионов Мо. На рис. 1 приведены гистограммы амплитуд ионных токов полевого испарения отдельных изотопов Мо при температуре эмиттера $T=1684 \mathrm{~K}$ для однозарядных ионов Мо, а на рис. 2 показаны такие же гистограммы для двухзарядных ионов. Получены они следующим образом. Прибор осуществлял примерно 200 сканов от массы 91 до массы 101 и детектор считал общее количество импульсов тока в секунду для всех 7 изотопов Мо. При этом величины импульсов были разными - от нескольких ионов до нескольких сотен ионов, так как процесс полевого испарения при высоких температурах носит принципиально нестационарный характер. Затем количество ионов каждого заряда для каждого изотопа усреднялось и определялось среднеквадратичное отклонение, которое и есть погрешность каждого такого измерения и, как правило, чем больше величина тока, тем больше и его флуктуации. Величины токов на рис. 1 и 2 указаны не в импульсах в секунду, как на следующем рис. 3, а в процентах от общего ионного тока. Как видно из этих графиков, наблюдаемое распределение всех 7 изотопов по массам как для однозарядных, так и для двухзарядных ионов примерно соответствует стандартному изотопному отношению для природного Мо. При этом в случае отдельных измерений токов отдельных изотопов могут, конечно, наблюдаться отклонения и даже значительные от природного распределения изотопов Мо (как для случая изотопа ${ }^{94} \mathrm{Mo}^{+}$на рис. 1), однако при наборе достаточно большой статистики подобные различия исчезают. Таким образом, значительный разброс данных, показанный в виде „усов“" на рис. 1 и 2, отражает неточность проведения эксперимента как такового, а соответствующий характер самого процесса высокотемпературного полевого испарения, когда наблюдается так называемый „эффект схлопывания колец“ - процесс периодического образования и испарения в виде ионов заметных кристаллических наростов на плотноупакованных гранях поверхности эмиттера, образующихся и испаряющихся вследствие процессов полевой диффузии, полевого кристаллического роста и полевого испарения [8]. По причине подобного процесса ионный ток носит пульсирующий характер даже при постоянном испаряющем поле [5,6].

Однако каким же способом мы можем определить для испаряющихся ионов Мо величину минимального расстояния для ионизации $-x_{\mathrm{cr}}$ ? Прежде всего для этого необходимо определить энергии образующихся в поле ионов. Ионы в ионном источнике вначале ускоряются до потенциала ускоряющего электрода $U_{a}=(10-15) \mathrm{kV}$, а затем замедляются до потенциала на коллекторе $U_{c}$. Если максимальная энергия иона в точности равна разности потенциалов между эмиттером и ускоряющим электродом, то $U_{c}=0$, величина $x_{\text {cr }}$ отсутствует и ионы образуются непосредственно на поверхности эмиттера, если же ионы рождаются на каком-то расстоянии от поверхности эмиттера, то энергии ионов будут меньше разности потенциалов на величину $U_{c}$ и чем дальше от поверхности рождается ион, тем больше будет ве- 


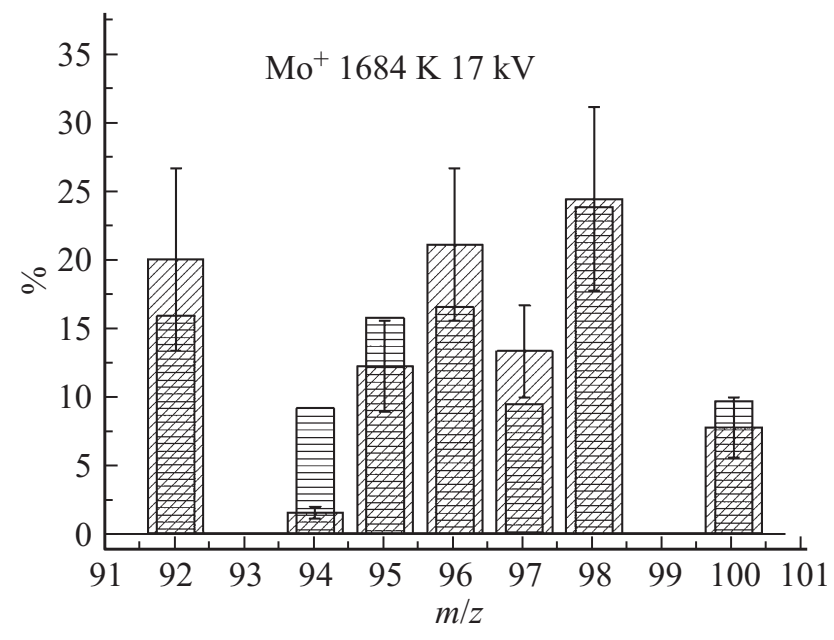

Pис. 1. Гистограммы ионного тока полевого испарения отдельных изотопов молибдена для однозарядных ионов $\mathrm{Mo}^{+}$ при температуре эмиттера $T=1684 \mathrm{~K}$ и $U=17 \mathrm{kV}$. Широкие столбики с косой чертой - экспериментально наблюдаемые ионы данной массы, узкие столбики с прямой чертой природное распределение изотопов по массам.

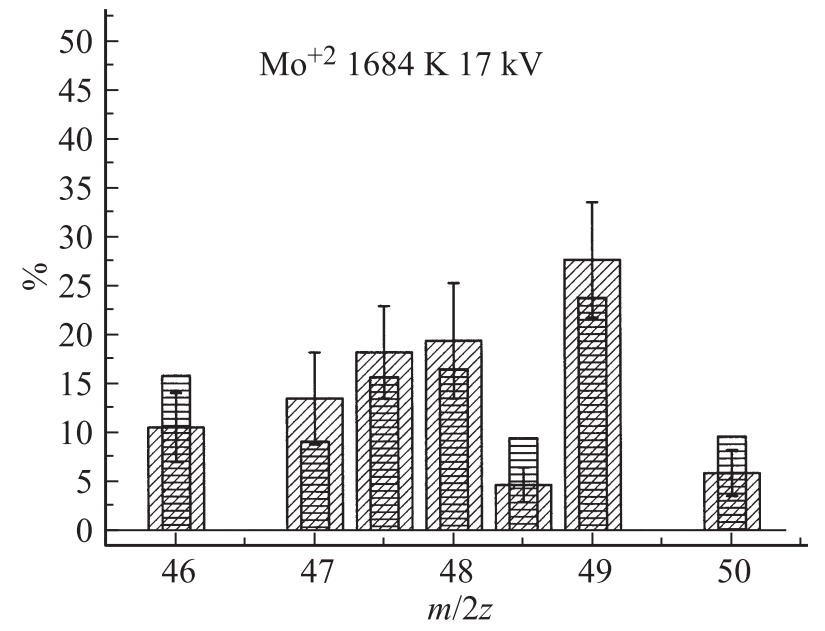

Pис. 2. Гистограммы ионного тока полевого испарения отдельных изотопов молибдена для двухзарядных ионов $\mathrm{Mo}^{+}$ при температуре эмиттера $T=1684 \mathrm{~K}$ и $U=17 \mathrm{kV}$. Широкие столбики с косой чертой - экспериментально наблюдаемые ионы данной массы, узкие столбики с прямой чертой природное распределение изотопов по массам.

личина $U_{c}$. После определения величины $U_{c}$ необходимо определить величину испаряющего поля $F_{\mathrm{ev}}$, при которой испаряется данный ион, и затем поделить эти величины.

На рис. 3 показано изменение тока ионов молибдена $i$ в зависимости от $T$. Сам по себе рост $T$ приводит только к экспоненциальному росту $i$ и не может влиять на заряд иона, сложный характер изменения величин $i$ ионов разных зарядов объясняется тем, что при одновременном воздействии высоких температур и сильных полей происходит сложное изменение формы острийного эмиттера и вследствие этого сложным образом меняется и величина $F_{\text {ev }}$ даже при неизменном приложенном напряжении $U_{a}$, а именно величина $F_{\text {ev }}$ и определяет заряд иона [5]. Если в случае испарения при комнатной температуре на спектрах наблюдались в основном ионы $\mathrm{Mo}^{+3}$, то рост $T$ приводил к заметному появлению ионов $\mathrm{Mo}^{+2}$ и $\mathrm{Mo}^{+}$, при $T \geq 1400 \mathrm{~K}$ основной пик обусловлен ионами $\mathrm{Mo}^{+}$. Видно, что при $T \sim 1400 \mathrm{~K}$ кривые тока пересекаются, что дает возможность определить величину $F_{\mathrm{ev}}$. Предполагаем, что и в данном случае зависимость скорости полевого испарения от $T$ характеризуется обычным законом Аррениуса, только величина энергии активации испарения зависит от приложенного поля. Согласно [2], полагаем, что величина ионного тока полевого испарения определяется выражением

$$
i=v_{0} \exp \left(-Q_{n} / k T\right)
$$

где $v_{0}$ - предэкспоненциальный множитель, а $Q_{n}-$ энергия активации испарения $n$-кратно заряженного иона. Выражение же для $Q_{n}$ удобно представить в следующем виде:

$$
Q_{n}=\lambda_{0}+\Sigma_{n} I_{n}-n \varphi-1.2 n \sqrt{n} F+3.45 \cdot 10^{-7} \alpha F^{2},
$$

где $\lambda_{0}$ - теплота испарения материала эмиттера, $I_{n}$ потенциал ионизации $n$-кратно заряженного иона, $\varphi$ работа выхода эмиттера и $\alpha-$ поляризуемость иона. При такой записи выражения величина $Q_{n}$ получается непосредственно в $\mathrm{eV}$, если $F_{\text {ev }}$ определяется в $\mathrm{V} / \mathrm{nm}$, а $\alpha-$ в $\mathrm{nm}^{3}$. Если токи одно- и двухзарядных ионов в точке пересечения равны, то равны и величины $Q_{n}$, т. е. $Q_{1}=Q_{2}$ и пренебрегая членом с поляризацией:

$$
F_{\text {ev }}=\left[\left(I_{2}-\varphi\right) / 2.19\right]^{2},
$$

подставляя соответствующие величины $I_{2}$ и $\varphi$, получим величину $F_{\mathrm{ev}}=29.6 \mathrm{~V} / \mathrm{nm}$, что заметно меньше величины $F_{\text {ev }}=46 \mathrm{~V} / \mathrm{nm}$ для случая испарения при криогенных температурах [1].

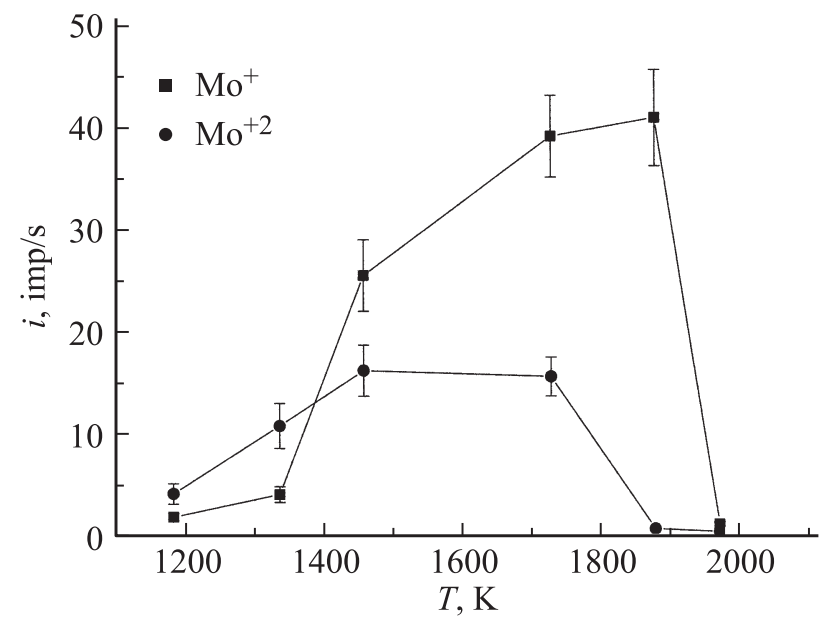

Рис. 3. Изменения токов $i$ ионов $\mathrm{Mo}^{+}$и $\mathrm{Mo}^{+2}$ в зависимости от температуры эмиттера $T$. 


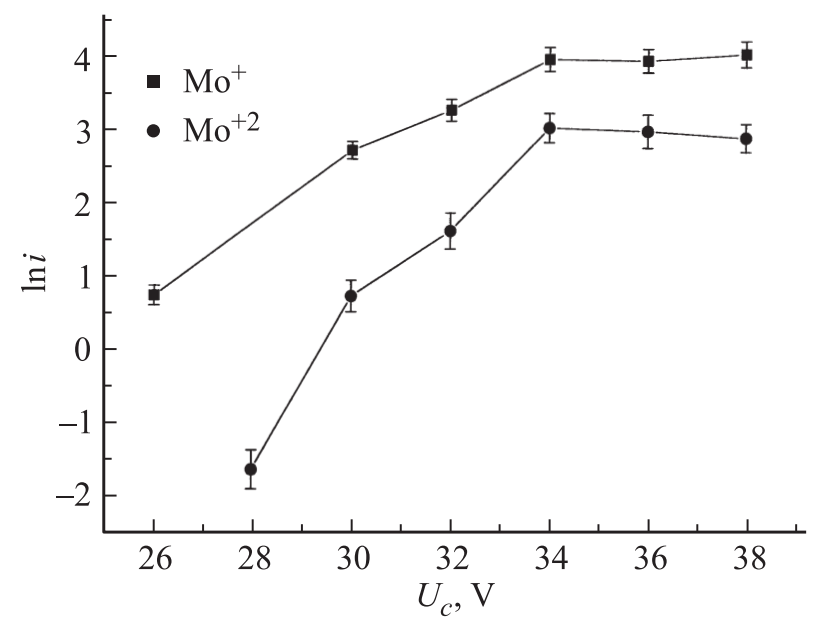

Рис. 4. Изменение логарифмов токов $i$ ионов $\mathrm{Mo}^{+}$и $\mathrm{Mo}^{+2}$ в зависимости от потенциала на коллекторе $U_{c}$.

На рис. 4 представлены зависимости изменения логарифмов тока ионов $\mathrm{Mo}^{+}$и $\mathrm{Mo}^{+2}$ в зависимости от потенциала на коллекторе $U_{c}$ (подобные кривые называют обычно кривыми задержки). Как уже отмечалось, если ионы рождаются непосредственно на поверхности эмиттера, то ионные токи должны наблюдаться при $U_{c}=0$. Однако рис. 4 показано, что величины $U_{c}$ существенно отличны от нуля и ток $\mathrm{Mo}^{+}$наблюдается в интервале $U_{c}$ от 26 до $34 \mathrm{~V}$, а для ионов $\mathrm{Mo}^{+2}$ наблюдается более узкий интервал $U_{c}$ от 28 до $34 \mathrm{~V}$. Таким образом, образование ионов происходит явно не на поверхности эмиттера, а где-то вблизи ее. Однако определить величины $x_{\mathrm{cr}}$, т. е. величины минимальных от поверхности расстояний, на которых образуются ионы, а также и ширины зон ионизации $\Delta$ на основании только полученных данных, не представляется возможным, поскольку величина $U_{c}$ может быть обусловлена также и различными приборными эффектами, как то „провисающими“ электрическими полями, контактными разностями потенциалов и др. Для устранения подобных возможных влияний параметров прибора и определения истинных величин $U_{c}$ был проведен в этом же ионном источнике эксперимент по поверхностной ионизации атомов цезия. На такой же, но вольфрамовый эмиттер была осуществлена адсорбция атомов $\mathrm{Cs}$, при этом хорошо известно, что атом Cs, адсорбированный на $W$, изначально уже является ионом [9], поскольку его потенциал ионизации $I_{1}=3.9 \mathrm{eV}$ заметно меньше средней работы выхода $W$, величина которой $\varphi=4.5 \mathrm{eV}[10,11]$. Поэтому если удалить адсорбированный ион Cs c поверхности $W$ легким прогревом и зарегистрировать этот ион на коллекторе источника ионов, то никакого дефицита энергии у такого иона быть не может и величина $U_{c}$ должна равняться нулю. Однако проведенный таким образом эксперимент по поверхностной ионизации цезия на вольфраме показал, что величина потенциала на коллекторе, соответству- ющего прохождению всего ионного тока $\mathrm{Cs}$, не равна нулю и составляет $U_{c}=11 \mathrm{~V}$. Таким образом, реальные величины $U_{c}$ для ионов $\mathrm{Mo}^{+}$и $\mathrm{Mo}^{+2}$ оказываются меньше измеренной величины на $11 \mathrm{~V}$ и реально составляют $U_{c}^{1}=(15-23) \mathrm{V}$ и $U_{c}^{2}=(17-23) \mathrm{V}$ соответственно. Определение истинных величин $U_{c}$ дает возможность при известной величине испаряющего поля $F_{\mathrm{ev}}$ определить и величины $x_{\text {cr }}$ и $\Delta$. Значения этих величин составляют для образования одно- и двухзарядных ионов $x_{\mathrm{cr}}^{2}=0.51 \mathrm{~nm}$ и $x_{\mathrm{cr}}^{2}=0.57 \mathrm{~nm}$ соответственно, а соответствующие значения для ширин зон ионизации будут для однозарядных и двухзарядных ионов $\Delta_{1}=0.27 \mathrm{~nm}$ и $\Delta_{2}=0.20 \mathrm{~nm}$.

О чем же говорят полученные величины $x_{\mathrm{cr}}$ и $\Delta$ ? Данные значения говорят о том, что, во-первых, в процессе высокотемпературного полевого испарения атомы не испаряются непосредственно с поверхности эмиттера в виде ионов, а образуются вблизи поверхности на расстоянии примерно двух атомных диаметров (диаметр атома Мо - $0.278 \mathrm{~nm}$ ) в очень узкой пространственной зоне шириной $\Delta \sim 0.20-0.27 \mathrm{~nm}$. Во-вторых, тот факт, что $x_{\mathrm{cr}}^{1}<x_{\mathrm{cr}}^{2}$, а также и $\Delta_{2}<\Delta_{1}$, скорее всего, подтверждает ранее высказанную точку зрения на процесс образования многозарядных ионов при криогенном полевом испарении, согласно которой вначале образуется однозарядный ион, который далее последовательно ионизуется с повышением заряда [12]. При этом если на рис. 1 наблюдается примерно $1.5 \%$ ионов ${ }^{94} \mathrm{Mo}^{+}$, a на рис. 2 содержание двухзарядных ионов этого же изотопа ${ }^{94} \mathrm{Mo}^{+2}$ составляет примерно $8 \%$, то это не означает, конечно, что $1.5 \%$ однозарядных ионов породили $8 \%$ двухзарядных, а просто сохранилось $1.5 \%$ первично образовавшихся однозарядных, после того как остальные испарившиеся ионы стали двухзарядными.

\section{Заключение}

1. Испарение Мо при высоких температурах происходит при низких величинах испаряющих полей $F_{\text {ev }} \sim 30 \mathrm{~V} / \mathrm{nm}$, тогда как при криогенных температурах величины $F_{\text {ev }} \sim 45-46 \mathrm{~V} / \mathrm{nm}$.

2. В случае полевого испарения при $T \geq 1000 \mathrm{~K}$ для всех 7 изотопов Мо наблюдаются только низкозарядные ионы $\mathrm{Mo}^{+2}$ и $\mathrm{Mo}^{+}$и чем выше температура, тем, как правило, больше доля однозарядных ионов в общем токе.

3. Превращение атома в ион в процессе высокотемпературного полевого испарения Мо происходит на расстояниях примерно два-три атомных диаметра от поверхности эмиттера в узкой зоне шириной примерно в один атомный диаметр.

4. Величины $x_{\mathrm{cr}}$ и $\Delta$ для ионов разной зарядности показывают, что, вероятнее всего, процесс образования многозарядных ионов происходит посредством первичного образования однозарядного иона, который затем последовательно ионизуется с повышением заряда. 
Работа частично поддержана грантом РФФИ № 14-08-00317-а главным образом в части модернизации системы сбора и обработки данных полевого магнитного масс-спектрометра.

\section{Список литературы}

[1] Мюллер Э.В., Цонь Т. // Автоионная микроскопия. М.: Металлургия, 1972. 360 с.

[2] Мюллер Э.В., Цонг Т.T. // Полевая ионная микроскопия, полевая ионизация, полевое испарение. М.: Наука, 1980. $217 \mathrm{c}$.

[3] Tsong T.T. // J. Chem. Phys. 1971. Vol. 54. P. 4205-4211.

[4] Бакулина И.Н., Блашенков Н.М., Лаврентьев Г.Я. и др. // А.c. № 711935. БИ. 1987. № 48.

[5] Голубев О.Л., Блашенков Н.М., Лаврентьев Г.Я. // Письма в ЖТФ. 2006. Т. 32. Вып. 20. С. 46-51.

[6] Голубев О.Л., Блашенков Н.М., Лаврентьев Г.Я. // ЖТФ. 2007. Т. 77. Вып. 10. С. 11-15.

[7] Голубев О.Л., Блашенков Н.М., Логинов М.В. // ЖТФ. 2012. Т. 82. Вып. 3. С. 111-116.

[8] Бутенко В.Г., Голубев О.Л., Конторович Е.Л., Шредник В.Н. // Письма в ЖТФ. 1992. Т. 18. Вып. 8. С. 86-91.

[9] Зандберг Э.Я., Ионов Н.И. // Поверхностная ионизация. М.: Наука, 1969. 430 с.

[10] Энергии разрыва химических связей, потенциалы ионизации, сродство к электрону. Справочник. М.: Наука, 1974. $351 \mathrm{c}$.

[11] Фоменко В.С. Эмиссионные свойства материалов. Справочник. Киев: Наукова Думка, 1981. 338 с.

[12] Kellog G.L. // Surf. Sci. 1982. Vol. 120. P. 319-333. 